\title{
Syringe-feeding as a novel delivery method for accurate individual dosing of probiotics in rats
}

\author{
S. Tillmann" and G. Wegener \\ Translational Neuropsychiatry Unit, Department of Clinical Medicine, Aarhus University, Skovagervej 2, 8240 Risskov, \\ Denmark;sti@clin.au.dk
}

Received: 27 August 2017 / Accepted: 9 November 2017

(c) 2017 Wageningen Academic Publishers

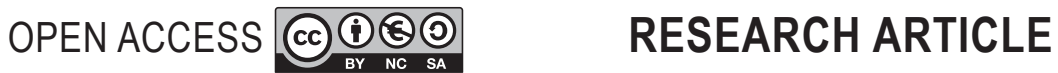

\begin{abstract}
Probiotic administration to rodents is typically achieved using oral gavage or water bottles, but both approaches may compromise animal welfare, bacterial viability, dosing accuracy, or ease of administration. Oral gavage dosing may induce stress, especially when given daily over several weeks, and cannot be performed by inexperienced personnel. Delivery in water bottles does not take multiple co-housed animals into account, leading to inaccurate dosing of individual rats. Moreover, slow consumption of the solutions over several hours may lead to variability in bacterial stability, and potential leftovers or clogging of the bottle further threaten the reliability of this method. To date, no method has been described that can provide non-stressful precise dosing of probiotics or prebiotics in individual rats. In accordance with the $3 \mathrm{R}$ principles (replace, reduce, refine), we propose syringe-feeding as a refinement method for simple yet accurate administration of probiotics. Animals hereby voluntarily consume the solution directly from a syringe held into their home cage, thereby enabling controlled dosing of individual animals. This method requires a short training phase of approximately 3 days, but is very fast thereafter, only taking seconds per rat. Since studies using probiotics are usually long-term experiments, we consider syringe-feeding the most appropriate probiotic delivery mode available to date.
\end{abstract}

Keywords: Lactobacillus, Bifidobacterium, lactic acid bacteria, synbiotics, drinking water

\section{Introduction}

The gut microbiota is increasingly recognised as a crucial mediator of human health (Marchesi et al., 2016), and several strategies have been employed to modify its bacterial composition. To this end, probiotics have shown promising efficacy for a range of disorders, in particular gastrointestinal indications (Ritchie and Romanuk, 2012). Recent studies also suggested a potential clinical use in extraintestinal diseases, such as type II diabetes (Wang et al., 2017), rheumatoid arthritis (Wang et al., 2016), Alzheimer's disease (Akbari et al., 2016), and depression (Wallace and Milev, 2017). However, clinical recommendations remain premature due to a lack of high-quality evidence (Rondanelli et al., 2017). To elucidate the potential therapeutic role of probiotics in greater detail, more preclinical studies using in vitro approaches or appropriate animal models are needed. Increasing the quality of such studies will separate the wheat from the chaff, and thereby facilitate informed clinical decision-making.

One of the most important quality demands is the controlled delivery of the candidate supplement into the host organism. Most studies use oral gavages or water bottles to administer probiotics to rodents. While oral gavage dosing does provide accurate dosing, it is time-consuming and potentially stressful for the animals, depending on the volume and vehicle administered (Brown et al., 2000). The use of water bottles imposes less strain on both animals and researchers, but is flawed due to uncontrolled dosing and time/speed of consumption. Rats are typically pair-housed, so multiple animals consume the probiotic solution from the same water bottle, thereby hindering correct dosing of each individual rat. Moreover, depending on the concentration and volume of the probiotics, it may take them several 
hours to finish the solution in the bottle, leading to potential variations in bacterial viability.

To ensure precise individual dosing, we propose syringefeeding as an alternative oral delivery method for probiotics. Syringe-feeding, voluntarily consuming a substance directly from a syringe, is well known in veterinary medicine, but is not widely used in research. To our knowledge, it has not been acknowledged as a potential method for probiotic delivery in rats, although probiotics are usually delivered in a sweet and palatable vehicle and are therefore suited for this approach. We hereby share the protocol used in our facilities to overcome current delivery problems and improve the quality of animal studies using probiotics.

\section{Materials}

\section{Animals}

We have successfully used this method with several rat strains, including Sprague Dawley (Taconic Bioscience A/S, Ry, Denmark), Wistar (Charles River, Sulzfeld, Germany), Flinders Sensitive Line (animal model of depression (Overstreet et al., 2005)), and Flinders Resistant Line rats (control strain for this animal model). Flinders Sensitive/ Resistant Line rats were bred at our facilities, but were originally derived from the colony at the University of North Carolina, USA. All our tested rats $(\mathrm{n}>150)$ were males aged between 7 and 21 weeks, approximately weighing 200-550 g. They were pair-housed in standard cages (Cage $1291 \mathrm{H}$ Eurostandard Type III H, $425 \times 266 \times 185 \mathrm{~mm}$, Tecniplast, Buguggiate, Italy) at $20 \pm 2{ }^{\circ} \mathrm{C}$ and $60 \pm 5 \%$ relative humidity on a $12 \mathrm{~h}$ light/dark cycle (lights on at $2 \mathrm{pm}$ ). All rats received water and standard chow ad libitum and had access to a tunnel shelter, nesting material, and a wooden stick. No animals were fasted or water-deprived at any time point. Handling is not necessary prior to the training phase, but may ease the process.

\section{Preparation}

Required materials are a towel, a standard single-use syringe, and the probiotic or vehicle solution sufficient for the desired amount of animals. To apply this method, the solutions need to be sufficiently palatable in sweetened vehicle. Most commercial formulations contain a polysaccharide food additive, such as corn-derived maltodextrin. We have used volumes ranging from 0.3 to $3 \mathrm{ml}$ per rat, and all rats have learned to voluntarily consume these. However, the smaller the volume, the more accurate the dosing will be, as the rat may otherwise not be able to consume everything in a single attempt, potentially leading to inaccuracies. A smaller volume may also make the solution more concentrated (i.e. sweeter) and therefore more palatable to the rat. We recommend a maximum volume of $1.5 \mathrm{ml} / \mathrm{rat}$, and typically treat with $0.3 \mathrm{ml}$.

\section{Procedure}

\section{Training phase}

The training phase is part of the experimental study, meaning that the probiotic/placebo formulations used in the intervention are also used during training. No other solutions are required for this method.

Remove the rat from its cage and place it on a towel on a table or your lap. Hold it with a gentle but firm grip and slowly release the solution into its mouth. The syringe should not enter the mouth cavity beyond its tip. Proceed in increments and occasionally pause to allow swallowing in between attempts. The rat may be returned to its cage for this purpose, although this is usually only necessary with large volumes. Alternating between two pair-housed rats will accelerate the process, but the consumed volumes should be controlled using two different syringes. Continue to feed the rat with the solution. Depending on the volume, this step may take anywhere from $20 \mathrm{sec}$ to $5 \mathrm{~min}$ per rat.

This procedure needs to be repeated until the rat gets accustomed to the taste. From our experience, this usually takes 3-4 days, but may differ between animals. During the first 2 days, the rat receives the probiotics or vehicle solutions while held on a towel outside the cage. From the third day, the rat may be held in its cage to facilitate the association of syringe-feeding with the home cage.

To test whether the rat is ready for the next phase, simply hold the syringe into the cage and observe if the rat approaches it and wants to drink from it. All of our tested animals $(n>150)$ have reliably reached this phase.

\section{Execution phase}

In this phase, the rat approaches the syringe readily and voluntarily consumes the solution, without the need to remove it from its cage. A photo of a successful feeding procedure during the execution phase is provided in Figure 1 and a video as Supplementary Information.

Prepare your probiotic or vehicle solution and draw up the required volume for the animals in the first cage. Locate the first rat and hold the syringe in front of its mouth. It should now readily drink the solution from the syringe. Keep track of the volume and make sure to memorise the animal that you fed first, then continue with the second animal. The syringe may be used for all animals within one treatment group. After all animals have been fed, simply discard the syringe. Our longest duration of the execution phase was 11 weeks, but extended treatment periods seem possible, as the animals did not show any signs of sudden aversion. 


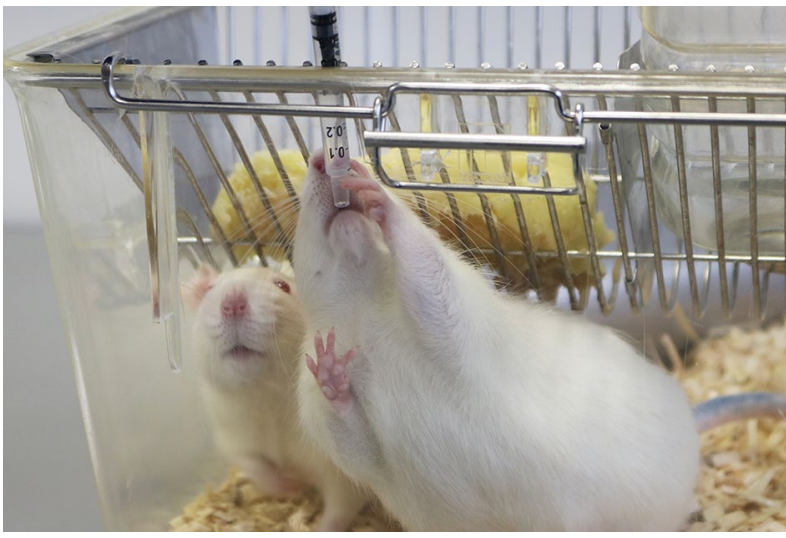

Figure 1. Rat voluntarily consuming probiotics from a syringe.

\section{Discussion}

This study aimed to adapt the syringe-feeding method for probiotic delivery in rats. We consider it superior to commonly used methods because it is accurate even with multiple animals per cage, easy to administer, fast, and presumably less stressful for the animals.

When choosing the optimal delivery mode for probiotics in rats, several criteria need to be met (Table 1). The welfare of animals should be the main ethical and scientific concern, as stress may introduce substantial bias (Lupien et al., 2009). Social isolation in single cages for the purpose of precise dosing should therefore be avoided. Oral gavage dosing involves potential stressors, such as removal from the cage, physical restraint, and the insertion of the gavage into the oesophagus. Moreover, there is a considerable risk for oesophageal damage, tracheal entry, and stomach distension (Balcombe et al., 2004), which may further be increased by researchers with less experience in animal husbandry, although this remains to be proven empirically.

A second criterion for precise administration is the stability of the probiotic bacteria. To our knowledge, no studies have investigated this in detail, but it seems likely that bacterial viability may vary between immediate delivery and delivery after several hours, as is usually the case for water bottles. Delivering probiotics in the drinking water of animals can be achieved in two different manners. In the first, a small and concentrated amount of the probiotics is given in a second bottle, in addition to the normal water bottle. Since the solution is concentrated and therefore usually palatable to the rat, it readily drinks the content of the bottle, circumventing any potential problems with bacterial viability. In the second manner, only one water bottle is present, which contains a more diluted concentration of the probiotics. Rats do not drink this solution immediately, potentially introducing variability in the bacterial stability. The severity of this delay needs to be addressed in future studies, but a time-controlled delivery is deemed preferable.

Accurate delivery is a third criterion essential to the correct interpretation of the results. Delivering probiotics with oral gavage is very precise, but the use of water bottles introduces several problems. Rats are usually co-housed due to their social nature, meaning that multiple animals consume the probiotic solution from the same water bottle. Precise individual dosing is therefore impossible, and it is generally assumed that an average value will suffice. However, one rat may be dominant over the other and consume more of the solution, which may lead to differences in response vs non-response to the probiotic treatment. Furthermore, it is difficult to estimate the consumed volume due to leftovers in the bottle. Potential clogging of the bottles and

Table 1. Overview of common methods used for chronic probiotic delivery in rats and their presumed advantages and limitations. ${ }^{1}$

Water bottles (concentrated)
Water bottles (diluted)
Oral gavage
Syringe-feeding


the aforementioned lack of control over the time and speed of consumption further threaten the experimental reliability and make the average intake an unreliable measure.

Ease of administration is another important criterion when choosing the delivery mode. Probiotic experiments are usually performed daily over several weeks. Therefore, a method that is fast and convenient is preferable. Water bottles need to be changed daily, which is inconvenient but usually does not take a large amount of time. Moreover, it can be done by inexperienced personnel, e.g. during the weekend. Oral gavage dosing requires proper animal handling skills and is time-consuming, since all animals need to be removed from their cages.

Syringe-feeding meets all four described criteria for optimal delivery. It does not seem to stress the animals; rather, they seem to enjoy consuming the probiotics, similar to concentrated water bottles with a small volume. Bacterial stability is conserved owing to the controlled time and temperature of delivery. Importantly, individual dosing of multiple rats per cage is possible, which is a significant advantage over water bottles and facilitates the collection of reliable data. Moreover, syringe-feeding in the execution phase (spanning over multiple weeks) can be performed by inexperienced personnel, which is an advantage for chronic studies. The delivery is very fast and only takes seconds for each rat, as demonstrated in the video (Supplementary Information). Moreover, syringe-feeding may be considered more translational (i.e. closer to human probiotic intake) than delivery with oral gavage. A comparison of the presumed advantages and limitations of commonly used methods for probiotic intake in rats is provided in Table 1. This comparison is intended to provide an overview and does not supersede empirical testing of different delivery methods, which should be provided by future studies.

Three caveats of the syringe-feeding technique need to be taken into consideration. First, it requires a 3-day training phase, which makes it less suitable for shortterm experiments. However, for chronic experiments, we recommend investing this time, since administration in the execution phase is very fast and convenient. Moreover, for this method to be successful, the probiotic and vehicle formulations need to contain sweetened food additives. Probiotics or prebiotics not containing a saccharide vehicle such as maltodextrin will not be palatable to the rats and therefore prevent voluntary consumption. However, this caveat also applies to bottle-feeding. Finally, syringe-feeding is best suited for small volumes $(<1.5 \mathrm{ml} /$ rat/day). Larger volumes are possible if spread over multiple time points, but may be more achievable with oral gavage or water bottles. However, aforementioned problems with both administration modes apply.
In conclusion, we recommend the use of syringe-feeding for probiotic delivery in rats because it is highly accurate, easy to administer, and animal-friendly. The 3Rs (replacement, reduction, and refinement; Russell and Burch, 1959) are the guiding principles for ethical use of research animals. In accordance with these standards, syringe-feeding may be considered a refinement strategy, as it may minimise potential pain or distress and thereby enhance animal welfare. The method itself has been described elsewhere (Atcha et al., 2010; Diogo et al., 2015), but, to our knowledge, it has not been adapted and used for research with pro-/ or prebiotics. Precise preclinical studies in this field are urgently needed to guide future clinical recommendations. We therefore wish to establish this method due to its prominent advantages and hope that other researchers find it useful for their purposes.

\section{Supplementary material}

Supplementary material can be found online at https://doi. org/10.3920/BM2017.0127.

Video showing the probiotic syringe-feeding procedure for rats.

\section{References}

Akbari, E., Asemi, Z., Daneshvar Kakhaki, R., Bahmani, F., Kouchaki, E., Tamtaji, O.R., Hamidi, G.A. and Salami, M., 2016. Effect of probiotic supplementation on cognitive function and metabolic status in Alzheimer's disease: a randomized, double-blind and controlled trial. Frontiers in Aging Neuroscience 8: 256.

Atcha, Z., Rourke, C., Neo, A.H., Goh, C.W., Lim, J.S., Aw, C.C., Browne, E.R. and Pemberton, D.J., 2010. Alternative method of oral dosing for rats. Journal of the American Association for Laboratory Animal Science 49: 335-343.

Balcombe, J.P., Barnard, N.D. and Sandusky, C., 2004. Laboratory routines cause animal stress. Contemporary Topics in Laboratory Animal Science 43: 42-51.

Brown, A.P., Dinger, N. and Levine, B.S., 2000. Stress produced by gavage administration in the rat. Contemporary Topics in Laboratory Animal Science 39: 17-21.

Diogo, L.N., Faustino, I.V., Afonso, R.A., Pereira, S.A., Monteiro, E.C. and Santos, A.I., 2015. Voluntary oral administration of losartan in rats. Journal of the American Association for Laboratory Animal Science 54: 549-556.

Lupien, S.J., McEwen, B.S., Gunnar, M.R. and Heim, C., 2009. Effects of stress throughout the lifespan on the brain, behaviour and cognition. Nature Reviews Neuroscience 10: 434-445.

Marchesi, J.R., Adams, D.H., Fava, F., Hermes, G.D., Hirschfield, G.M., Hold, G., Quraishi, M.N., Kinross, J., Smidt, H., Tuohy, K.M., Thomas, L.V., Zoetendal, E.G. and Hart, A., 2016. The gut microbiota and host health: a new clinical frontier. Gut 65: 330-339.

Overstreet, D.H., Friedman, E., Mathe, A.A. and Yadid, G., 2005. The flinders sensitive line rat: a selectively bred putative animal model of depression. Neuroscience and Biobehavioral Reviews 29: 739-759. 
Ritchie, M.L. and Romanuk, T.N., 2012. A meta-analysis of probiotic efficacy for gastrointestinal diseases. PLoS ONE 7: e34938.

Rondanelli, M., Faliva, M.A., Perna, S., Giacosa, A., Peroni, G. and Castellazzi, A.M., 2017. Using probiotics in clinical practice: where are we now? A review of existing meta-analyses. Gut Microbes 22: 1-23.

Russell, W.M.S. and Burch, R.L., 1959. The principles of humane experimental technique. Universities Federation for Animal Welfare, Wheathampstead, UK.
Wallace, C.J. and Milev, R., 2017. The effects of probiotics on depressive symptoms in humans: a systematic review. Annals of General Psychiatry 16: 14.

Wang, P., Tao, J.H. and Pan, H.F., 2016. Probiotic bacteria: a viable adjuvant therapy for relieving symptoms of rheumatoid arthritis. Inflammopharmacology 24: 189-196.

Wang, X., Juan, Q.F., He, Y.W., Zhuang, L., Fang, Y.Y. and Wang, Y.H., 2017. Multiple effects of probiotics on different types of diabetes: a systematic review and meta-analysis of randomized, placebocontrolled trials. Journal of Pediatric Endocrinology and Metabolism 30: 611-622. 
